\title{
Narrative Exposure Therapy for the Treatment of Traumatized Children and Adolescents (KidNET): From Neurocognitive Theory to Field Intervention
}

Frank Neuner, $\mathrm{PhD}^{*}$, Claudia Catani, $\mathrm{PhD}$, Martina Ruf, MA, Elisabeth Schauer, $\mathrm{PhD}$, Maggie Schauer, $\mathrm{PhD}$, Thomas Elbert, $\mathrm{PhD}$

Department of Psychology, University of Konstanz, Box D25, D-78457 Konstanz, Germany

\section{Current evidence and practice}

Violence against children, including maltreatment at home, sexual abuse, and child labor, are common phenomena worldwide. In addition, many children are affected by violence caused by political conflicts, unrest, and war. Current wars are characterized by high levels of deliberate and systematic violence against the civilian population [1], including victimization of women and children. More than 30 countries are affected by current wars, and the populations of many now peaceful countries suffer from the aftermaths of a recent armed conflict. In addition, the number and intensity of environmental disasters is increasing. Environmental disasters mainly affect low-income countries with poor facilities for an adequate emergency response [2]. In some regions, war and environmental disaster co-occur [3], causing severe acute emergencies and a long-lasting mental health impairment in the population.

Although children can be astonishingly resilient even in face of severe disasters and atrocities, high levels of traumatic events increase the risk of developmental difficulties and psychologic disorders. Many studies have shown increased rates of mental disorders in children affected by war [4].

This work was supported by the Ministry of Science, Research and the Arts of BadenWürttemberg, the Deutsche Forschungsgemeinschaft, and the European Refugee Fund.

* Corresponding author.

E-mail address: frank.neuner@uni-konstanz.de (F. Neuner). 
The most prevalent disorder is posttraumatic stress disorder (PTSD), which consists predominantly of intrusive thoughts, flashbacks, nightmares, avoidance behavior, and increased levels of arousal. The prevalence of PTSD in children in war-affected populations ranges from $20 \%$ among Lebanese children who were exposed to bombings and terror attacks [5] to $44 \%$ in surviving orphans 10 years after the Rwandan genocide [6]. A survey in the war-affected northeastern region of Sri Lanka showed that $19 \%$ to $25 \%$ of the Tamil children already were suffering from PTSD before the region was hit by the devastating tsunami flood wave in 2004 [7]. After the tsunami, PTSD rates of $40 \%$ were found in communities affected by both war and disaster [8]. In addition, Catani and co-workers [3] found a high level of family violence in these communities. Confirming a general dose-effect model [9], PTSD in children was predicted mainly by the cumulative exposure to trauma including war, disaster, and family violence in this population.

Longitudinal studies show that trauma symptoms in children are more than simply a behavioral problem that dissipates over time as the child matures. Rates of spontaneous remission in traumatized children are remarkably low [10]. In a large proportion of severely war-traumatized children, PTSD can persist for more than 10 years even when the children are living in a safe environment [11]. As a consequence, intervention strategies for severely traumatized children in war-affected populations are necessary to prevent a downward spiral in development. Intervention research in this area is still in its infancy, however, and common practice rarely is informed by scientific knowledge. Even though regions in crisis and refugee camps often attract humanitarian workers from many different countries, psychosocial activities are restricted mostly to interventions such as creative play therapy, the indiscriminate distribution of psychoeducational material, and supportive counseling. Most often, the interventions provided by humanitarian workers and health professionals have been developed ad hoc without a solid theoretic background, and the efficacy of these methods, including play activities, is doubtful [12].

In recent years, knowledge about the treatment of PTSD in children living in high-resource countries has increased rapidly. There now is considerable evidence supporting different variants of cognitive behavioral therapy (CBT) for the treatment of school children who have PTSD resulting from multiple traumatic events, including sexual abuse [13] and other forms of violence [14]. In general, CBT programs include psychoeducation, cognitive interventions, training of affective coping skills, and exposure therapy. Several randomized trials have shown that in treating PTSD symptoms CBT is more effective than standard care, unspecific therapy, or no treatment [15]. Follow-up studies have demonstrated that treatment gains are maintained for up to 2 years [16].

Unfortunately, no randomized controlled study has included child war victims or traumatized refugees. Some pragmatic treatment programs 
have been developed for use in crises in low-resource countries or in refugee communities. For example the "Children and War: Teaching Recovery Techniques" approach [17] was developed as a trauma-focused group intervention for the context of disaster, war, and refugee populations and was tested in several uncontrolled and nonrandomized controlled studies. In general, trauma-focused group programs showed promising effects with significant reductions of PTSD symptoms [18]. Although clinically significant treatment effects could be demonstrated for disaster victims in uncontrolled studies $[19,20]$ and even in a randomized trial [21], treatment gains were modest and did not seem to be stable over time when applied to refugee children in the United Kingdom [22], and no significant effect was found for refugee children in Gaza [23].

The authors have developed narrative exposure therapy (NET) as a pragmatic, short-term intervention for traumatized victims of war and torture. NET is an individual-level, trauma-focused treatment that, on the one hand, builds on the tradition of testimony therapy developed by Lira and Weinstein to treat the victims of the Pinochet regime in Chile [24]. The key aspect of testimony therapy is the detailed documentation of human rights violations experienced by the survivor of political violence and the use of this testimony for political purposes. On the other hand, NET builds on the principles of current neurocognitive theories of PTSD and cognitive behavioral therapy by adapting the classical form of exposure therapy to meet the needs of traumatized survivors of war and torture. In exposure therapy for PTSD [25], the patient is asked to talk repeatedly about the worst traumatic event in detail while re-experiencing all emotions associated with the event. In the process, most patients undergo habituation of the emotional response to the traumatic memory. This habituation consequently leads to a remission of PTSD symptoms. Typically, victims of organized violence have experienced multiple traumatic events; the authors encountered a child who had seen her brother die from shrapnel wounds and who later was raped. As outlined later in this article, multiple traumatic events can cause a substantial distortion in autobiographic memory with an excessive emotional representation that includes an unsorted batch of stimuli from different memories. It therefore is difficult to differentiate different episodes or to identify a single worst event before treatment. Consequently, in NET, the patient constructs a narration of his or her whole life from early childhood to the present date while focusing on the detailed report of traumatic experiences [26].

\section{Theoretical background: neurocognitive memory theory}

The most prominent neurobiologic and cognitive theories of PTSD explain the development of PTSD on the basis of a pathologic distortion of the memory representations of the traumatic event [27-30]. According to Tulving [31], there is a specific store of memories about past events called 
"episodic memory." Episodic memory involves happenings in particular places at particular times and covers context information about "what," "where," and "when." A unique feature that distinguishes episodic memory from other memory systems is the possibility of consciously re-experiencing previous events in the form of recollective experiences. Most theories of episodic memory separate at least two different bases of episodic knowledge: nondeclarative memory and declarative memory (for a different view see [32]). These memory systems differ in the retrieval of information: declarative (explicit) memory can be retrieved deliberately and accessed verbally, whereas nondeclarative (implicit) memory is activated automatically by environmental or internal cues and affects a person's behavior and experience [33]. Therefore one must distinguish between a declarative episodic memory system (later called "autobiographical representation") and a nondeclarative episodic memory system (later called "sensory-perceptual representation"). In addition, there is good reason to assume that a supervisory structure controls the activity of the memory representations.

\section{Autobiographical representation}

The declarative part of episodic memory has been called "autobiographical memory" [34]; other authors have used the terms "verbally accessible memory" [35] or "cold memory" [36]. Autobiographical memory is a highly developed and structured memory system that allows the abundant knowledge about past events to be filed in a highly efficient way. Autobiographical memory is the principal resource for the retrieval of information about one's life and is the main base for the narration of events and life periods. To allow a rapid and organized access of information, this memory is structured in a hierarchical way. At the top of the organization are the lifetime periods that have identifiable beginnings and endings. They represent general knowledge of important other persons, locations, actions, activities, plans, and goals. An example of a lifetime period is "when I was living with Maria" or "when I was working at the farm." One level below the lifetime periods is the memory for general events. General events can be divided into repeated events (eg, "having lunch at the canteen") and specific events (eg, "my first day in school"). These knowledge bases organize the chronologic sequence of single events.

The neurobiologic correlate of autobiographical memory cannot be identified as a single brain structure. Rather, long-term storage of autobiographic and other declarative knowledge depends on widespread neocortical neuronal activity. This complex neuronal network underlies a special organization including specific rules and consistencies. This organization allows the effective storage of the vast amount of knowledge a human being can acquire. At the same time, this complex organization makes this network slow to integrate new incoming information, especially new events that are incompatible with previous knowledge. 
The key brain structure related to autobiographic memories is the hippocampus. The hippocampus itself is not the permanent store of declarative memory, but it has a central function in the consolidation of memory within the first 4 weeks of the event. In this period, the information is located to a wide neocortical network [37]. Several neuroscientists assume that the function of the hippocampus is to construct a meaningful episodic representation of the dynamics of the situation in a spatiotemporal context [38]. McClelland and coworkers [39] suggested that the hippocampus is especially important for the coding of information that contradicts previously learned knowledge. They assume that the neocortical system is slow to integrate contradictory information, because that information undermines strict rules and consistencies. In this context, the hippocampus may permit the formation of a rapid representation of the event and gradually expose the neocortical system to the new information. In this way the network can be reorganized slowly to integrate the new information.

\section{Sensory-perceptual representation}

When a person thinks about a past life event, it is be possible that he or she both retrieves abstract knowledge about what has happened and also can imagine the event in the form of a recollective experience [31]. Thus, in addition to declarative autobiographic facts, people can access directly the sensory and emotional information about past events stored in their minds, including an awareness of the subjective time when the event happened. The retrieval of this sensory information is fundamentally different from the retrieval of autobiographic information. Whereas the contextual facts stored in autobiographic memory are retrieved as verbally accessible knowledge, the retrieval of sensory information is perceived as an experience of the information itself. For example, a person who has a vivid memory of his first day at a beach actually might see the water in front of his eyes and re-experience the excitement of that moment when thinking back on the event. This type of information is provided by a sensory-perceptual representation of the event (other authors have used such terms as "hot memory" [36], "situationally accessible memory," [35] or "event-specific knowledge" [34]). The sensory-perceptual representation itself does not contain any context information about single events, but a close tie to the corresponding autobiographical representations offers a spatial and temporal context for this memory. As a consequence, the activation of the sensory-perceptual details of an event usually is accompanied by the activation of autobiographical knowledge about the sequence of the event and the location of the event in lifetime periods.

Obviously, a vivid and detailed recollection is not possible for all events experienced in a life. For everyday events that have a minor meaning for the person involved, the sensory-perceptual representations usually only last minutes or hours [40]. The enduring storage of sensory-perceptual 
representations happens only for events stored in a highly emotional state, because they are significant for the achievement or failure of a person's individual goals [34].

Lang's [41] bio-informational theory of emotions offers a good framework for understanding the nature of sensory-perceptual representations and their relationship to emotions. In this view, emotions are represented mentally as associative networks. These representations consist of sensory information about the stimuli present in the past situation in different modalities (eg, visual, auditory, olfactory). At the same time, this network contains information about the cognitive and affective evaluation of the stimuli and the corresponding physiologic responses. Fig. 1 provides a sensory-perceptual representation of a person's memory of a special moment on a beach. The items of these networks are connected so that the activation of single items causes the spreading activation of connected items. Items of the representation can be activated either by external stimuli that share features of the memory (eg, being at the sea again) or by thinking deeply about the event. A recollective experience of the memory can follow, because single sensory elements are interconnected and therefore are likely to be activated together. In addition, the recollective experience can be accompanied by the vivid sensation of the emotions, cognitions, and physiologic responses represented in the memory, because they are associated with the sensory elements. While remembering that day, the person actually might feel the cold water, and his heartbeat actually might increase.

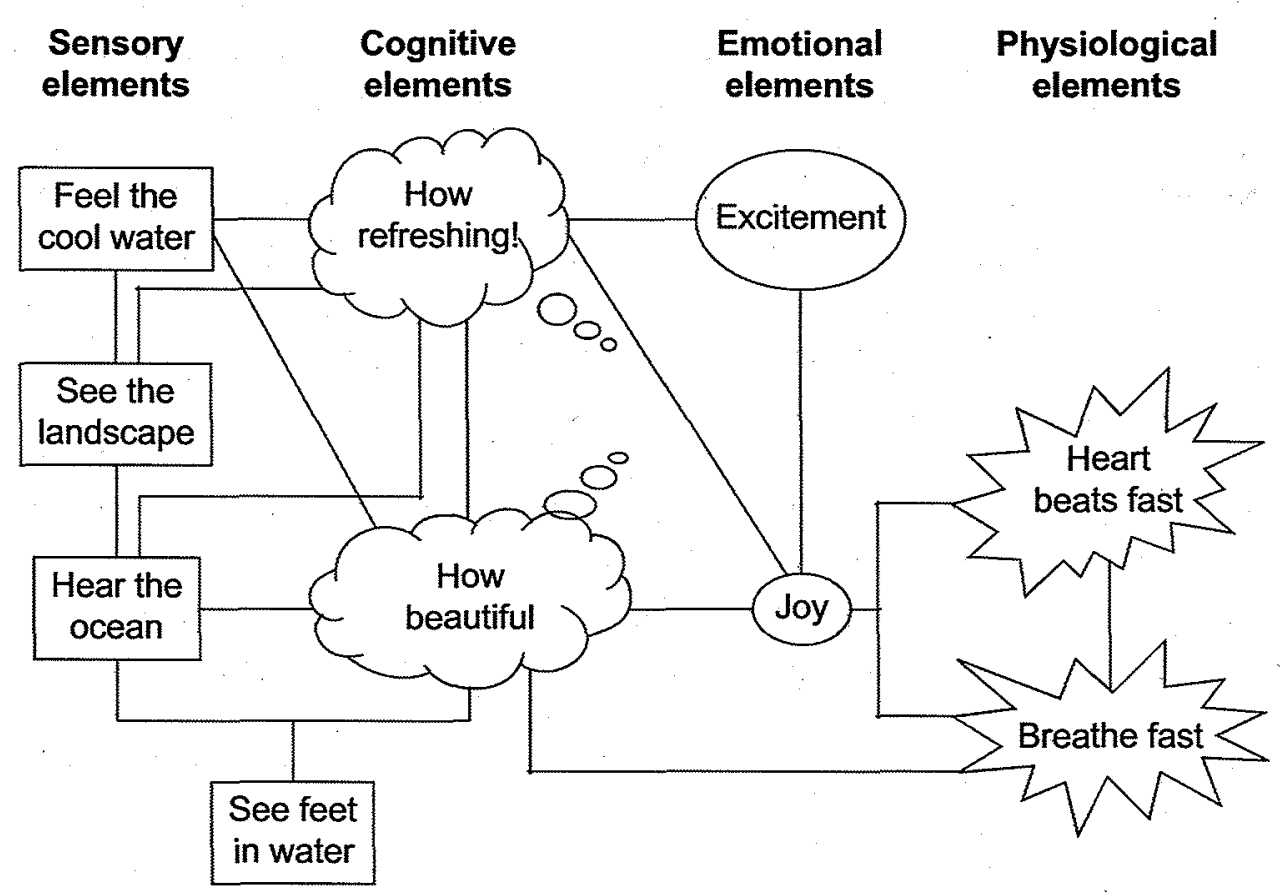

Fig. 1. Schematic presentation of a hypothetical sensory-perceptual representation including sensory, cognitive, emotional, and physiologic elements. The network represents a child's memory of a day on a beach during a holiday. 
Like autobiographic representations, the sensory-perceptual representations of past events probably are not stored in a single brain structure. Neuroimaging studies suggest the complex involvement of the areas considered responsible for visuospatial processing and emotion (the limbic structures, especially the posterior cingulated, including the surrounding cortex and the occipital and parietal cortex) and for preparation for action (motor cortex) in the storage of sensory-perceptual representations [42].

There is good evidence that the amygdala plays a key role in the encoding and retrieval of highly arousing events, whether positive (exciting) or negative (frightening) [43,44]. Research on "flashbulb memories" [45] contributed to the knowledge about sensory-perceptual memory representations. Flashbulb memories are vivid and long-lasting memories of photographic quality about the environmental conditions of a person learning of a significant public event such as the terrorist attacks of September 11, 2001. The elevated arousal in this situation seems to support the encoding of a lasting sensory-perceptual representation of the situation. Recently it was shown that the amygdala is a key structure for the recall of flashbulb memories related to the September 11, 2001 terrorist attacks, at least for the persons who had been close to the event itself [46]. These findings fit well with animal research on fear conditioning, showing that the amygdala is the key structure for the learning associations between sensory stimuli and fear responses $[47,48]$. The effects of fear conditioning may be based on sensory-perceptual representations of the learning situation. Because the sensory and cognitive outputs of these memories cannot be studied with animal research, researchers have concentrated on the physiologic and behavioral responses to conditioned fear cues.

\section{The effects of trauma on memory}

Traumatic events are characterized by a massive threat to the victim requiring the immediate activation of the body in the form of an alarm response. Within seconds, the brain triggers the secretion of stress hormones, including the catecholamines norepinephrine and epinephrine. In combination with other immediate effects of sympathetic nervous activation, this response prepares the body system for a rapid fight-or-flight response. More slowly, peaking around 20 minutes after this rapid reaction, the hormonal messenger corticotropin, secreted by the pituitary gland, has traveled to the adrenal cortex, which releases the stress hormone cortisol that modulates a series of additional functions, including an increase in blood sugar level. The stress hormones travel back to the brain where they dock on receptors that are particularly dense in the hippocampus and dramatically modulate memory formation and consolidation [49]. In general, exposure to glucocorticoids (the stress hormone in rodents corresponding to human cortisol) increases the activity of the hippocampus, but the activity declines dramatically as soon as a certain threshold is exceeded. Under 
very high levels of stress and under chronic stress, the functioning of the hippocampus is impaired severely. At least in rodents [50,51], but probably also in primates [52], very high doses of adrenal steroids can cause permanent atrophy of the hippocampus.

Unlike the hippocampus, which shows decreased functioning under high levels of stress, the activity of the amygdala is enhanced as stress increases [53]. This enhanced activity also is caused by stress hormones [54,55]. A recent study with rats showed that whereas chronic stress induced dendritic atrophy in the hippocampus, the dendritic arborization of neurons in the amygdala was enhanced in the same condition [56]. The dissociation of amygdala and hippocampal functions during high levels of stress could be confirmed in humans. Using functional MRI and simultaneous skin conductance response measures, Williams and colleagues [57] demonstrated that amygdala networks were active only during the elaboration of arousing stimuli, whereas hippocampus activity occurred only in the absence of arousing stimuli.

The stress effects on amygdala and hippocampus affect the memory representations of the traumatic events. On one hand, the increased amygdala activity leads to an excessive sensory-perceptual representation of the event that has also been called "fear structure" [58]. As a result of the excessive amygdala activity during extreme stress, a fear structure differs from representations of normal events in several ways. First, the fear structures encoded during a traumatic event are unusually large and cover a wide variety of single items. As a consequence, they can be activated easily, because many environmental cues resemble the items in the fear structure. Second, interconnections between the single elements are unusually strong. Fig. 2 shows an example of a fear structure of a Sri Lankan child who has experienced both war and tsunami. As shown in the figure, multiple traumatic events can become part of a single fear structure because they share common elements like fear and physiologic arousal.

Because of the strong interconnection of items, the activation of only a few elements is sufficient to activate the whole structure, and this activation is difficult to control. The activation of the fear structure is experienced as an intrusive sensory, emotional, and physiologic re-experiencing of the traumatic event, the key symptom of PTSD. As a consequence, traumatized persons learn to prevent the activation of the structure by avoiding cues that remind them of the traumatic event. They must avoid both internal and external cues, so they try not to think about the even, try not to talk about it, and try to keep away from persons and places that remind them of the event. Eventually, the person acquires a pattern of avoidance behavior, another characteristic of PTSD.

Whereas extreme stress causes an excessive sensory-perceptual representation of the traumatic event, stress impairs the functioning of the hippocampus and the elaboration of an autobiographical representation. As a result, the traumatic event does not seem to be clearly represented as 


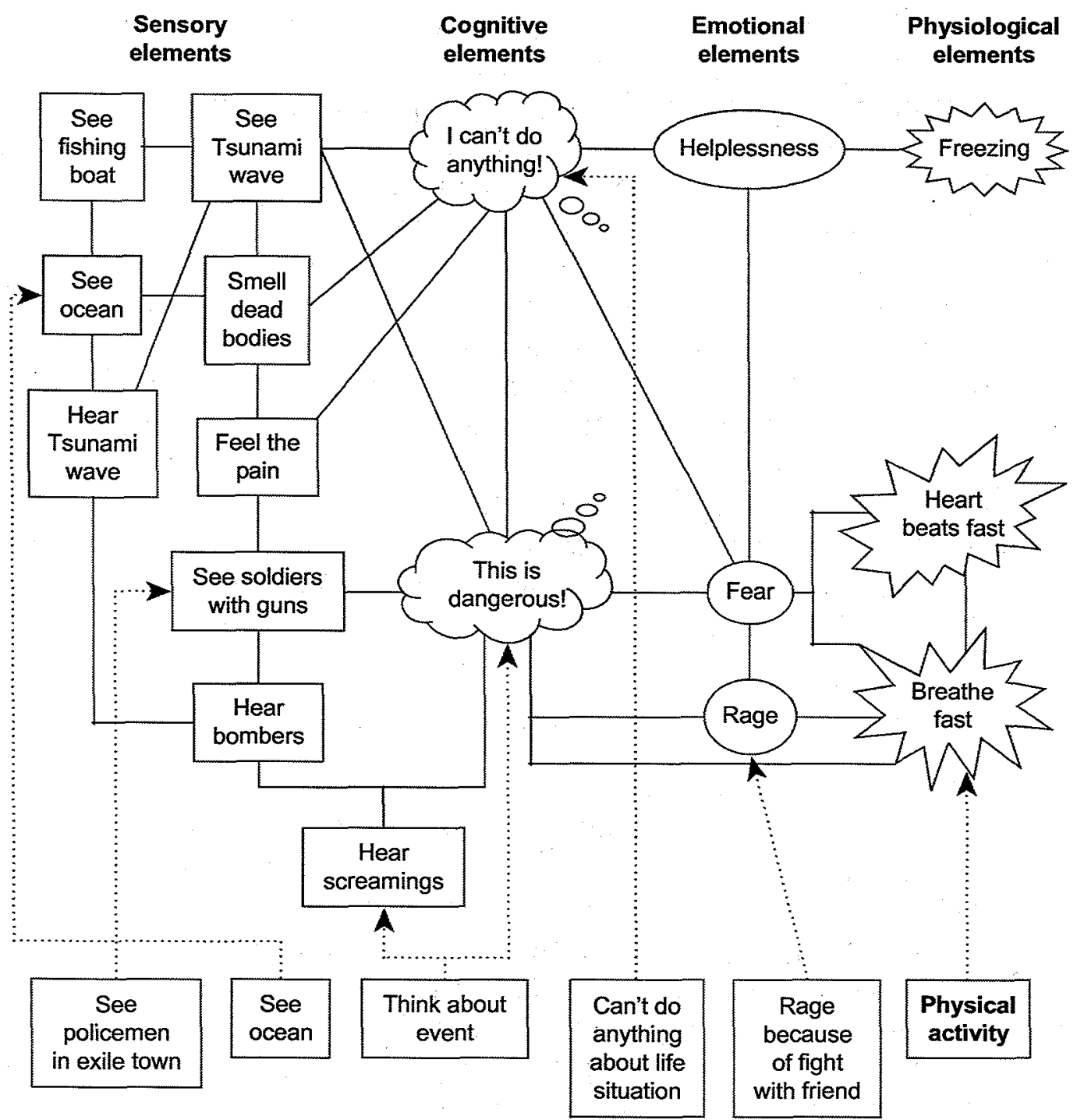

Fig. 2. Schematic presentation of a hypothetical sensory-perceptual representation of a traumatic event (fear structure) of a girl from Northern Sri Lanka. The network represents the memory of the Tsunami disaster mixed with war memories. The boxes below indicate environmental stimuli with the potential to activate the representation.

a specific event, and it does not seem to be clearly positioned in a lifetime period. Because a good autobiographical organization is necessary to narrate an event, the distortion in autobiographical memory leads to the failure of patients to talk about their traumatic experiences. In addition, the antagonism of the declarative and nondeclarative memory representations causes a distorted quality of retrieval of traumatic memory. The recollection of normal events usually is accompanied by the perception of subjective time, which means that even during the in-depth mental imagery of a past event a person always feels that this event took place at a different time and place. In contrast, the mostly intrusive recollection of traumatic memory is ungrounded by the narrative and spatiotemporal contextual anchors 
that tie ordinary experience to reality. Traumatic memories are disturbing, because of the direct fear they evoke and also because of their strangeness [36]. Lacking the autobiographical context, the intrusive memories are accompanied by the sensation of current threat, even though the event might have happened several years ago at a place very far away [30].

Taken together, the characteristic of traumatic memory is twofold. On the one hand, a person has very vivid recollections, including many sensory details. Despite the detailed representation of the memory, however, it is very difficult for the victim to narrate the event. Consistent with this assumption, several studies have shown that traumatic memory is qualitatively different from everyday memory [59]. Traumatic memory is more dominated by sensory elements and is highly distressing and repetitive, whereas the narrations are more fragmented [60]. In addition, Jones and co-workers [61] showed that disorganization of the narration and a dominance of sensory elements in the trauma memory immediately after the event predict the development of chronic PTSD.

\section{Recovery from trauma: role of the supervising structure}

Although most people react with an intensive emotional upheaval, including intrusive symptoms, immediately after a traumatic event, only a minority develops chronic PTSD. The recovery from acute stress symptoms is a normal process. In an influential account of emotional processing, Foa and Kozak [58] suggested that emotional processing involves the modification of the original fear structure. Research into fear conditioning has challenged this view. Several investigators have shown that extinction of fear responses usually does not change the original stimulus-fear associations, because the original fear response can be reinstated easily in a context different from the context of extinction learning. Instead, extinction probably occurs through the inhibition of the fear response by cortical areas, especially the medial prefrontal cortex [48]. Pathways from the prefrontal cortex to the amygdala allow the modification of a fear response, depending on the evaluation of the stimulus in the current environmental context. Recently, Shin and colleagues $[62,63]$ showed that during symptom provocation (script-driven imagery), as well as during unspecific emotional stimulation, patients who had PTSD showed decreased activity in the medial prefrontal region but increased amygdala activity. In addition, amygdala activity correlated negatively with medial prefrontal activity and correlated positively with symptom severity. These findings indicate that persons who recovered from trauma symptoms can rely on an effective prefrontal mechanism to inhibit the activity of the amygdala-related fear structure. Animal research supports this view, because fear extinction depends on the activity of medial prefrontal areas rather than on changing amygdala-dependent associations [64-66], and sustained fear extinction requires memory consolidation in prefrontal areas involving protein synthesis [67]. 
Brewin [27] suggested that recovery from trauma symptoms depends on the availability of declarative autobiographical memory of the stimulus. This contextual knowledge is necessary to identify the intrusive flash of a fear structure after a trauma reminder as a memory process relating to past event rather than an indication of current threat. After a traumatic event, however, declarative knowledge, especially autobiographical knowledge that could offer information about the context of the feared stimulus, is fragmented or even absent. In this conceptualization, recovery from initial intrusive symptoms after a stressful event is the result of active emotional regulation rather than automatic extinction of fear responses. Recent imaging studies have shown that activity of the prefrontal brain structures correlate with active emotion regulation (eg, cognitive re-evaluation) [6870] and with the inhibition of amygdala activity [69]. Furthermore, these structures overlap with areas responsible for extinction of conditioned responses [71]. These structures are key candidates for a supervisory structure that can control the activity of a fear structure, depending on the evaluation of the current situation and the memory content.

\section{Traumatic memory in children}

Episodic memory begins at the age of about 2 years, as soon as the children acquire a cognitive sense of self [72]. Theories of cognitive development suggest that early episodic memory is dominated by nondeclarative knowledge and contains little elaborate information about context and meaning [73]. Whereas sensory-perceptual representation can be established very early in life, fully functioning autobiographic memory depends on the development of a variety of cognitive functions, including mental concepts, temporal concepts, self-representations, language representations, and narrative structure [74]. In healthy children most of these structures have evolved sufficiently at the age of 5 years [32,74]. Because traumatic stress can affect a variety of brain structures during development [75], it is reasonable to assume that development of episodic memory may be delayed in children who grow up in stressful conditions. As a consequence, the memory theory outlined previously cannot be adapted unconditionally to children below the age of 5 years. In addition, the phenomenology of symptoms in traumatized preschool children differs to some extent from that in older children [76], and approaches to treatment must be adapted for these children [77].

Because of ethical concerns, there is a lack of research on the neurophysiology of trauma in children. In particular, there is no symptom-provocation study involving children. Some findings on traumatic memories do correspond with the neurocognitive theory, however. Recently, Kenardy and co-workers [78] examined children's trauma narratives in the aftermath of a traumatic event (4-7 weeks) and the presence of PTSD symptoms 6 months later. The authors found that temporal disorganization, but not absence of 
emotion, was associated with the presence of PTSD symptoms at a later stage. On the other hand, dissociative themes in the trauma narratives showed a weak link with the development of subsequent PTSD. These outcomes support the notion that in children, as well as in adults, traumatic memories might be characterized by a lack of coherent autobiographic information and, at the same time, by a dominant network of sensory and emotional elements.

In a recent longitudinal study [79], children who had a history of maltreatment underwent a variety of clinical evaluations at different time points over a period of 12 to 18 months. The study showed that the severity of PTSD symptoms as well as cortisol levels at baseline predicted hippocampal reduction. Even though additional studies are warranted to confirm these pilot data, the results of this study suggest an association among hippocampal changes, PTSD symptoms, and cortisol levels in traumatized children.

\section{Consequences for treatment}

In accordance with the neurocognitive theory of PTSD, the main goal of therapy could be the construction of a consistent declarative (autobiographic) representation of the traumatic event. Immediately after the fear structure has been activated by a reminder of a traumatic event, this declarative representation can provide the supervisory structure with the information that the activity of the fear structure is only a memory recollection and does not indicate a current threat. In turn, the supervisory structure can inhibit the activity of the fear structure. Consistent with this assumption, research on trauma treatment indicates that the construction of declarative trauma memory seems to be important for successful treatment. The exposure therapy developed by Foa and colleagues [25] has proven to be one of the most successful treatment approaches for PTSD. In exposure therapy for PTSD, the patient is instructed to talk about the traumatic experience repeatedly. Initial formulations of the theory of exposure therapy were based on fear extinction, but recent analyses of the treatment process support the importance of constructing autobiographic knowledge. In particular, it has been demonstrated that patients who manage to construct a coherent narration of the event during exposure therapy profit most from treatment [80].

The main focus of therapy should be on the part of the memory that is most fragmented in autobiography and is represented most intensively within the sensory-perceptual representations. To form a consistent autobiographic narration of this moment, the sensory-perceptual representation inevitably is activated, because it provides detailed knowledge about the event that is not yet available in declarative structures. The autobiographic representation should cover the most salient stimuli of the sensory-perceptual representation with the highest probability of 
eliciting intrusive symptoms. Because the activation of the sensory-perceptual representation always is accompanied by the emotional reactions coded in this structure, a high emotional involvement is necessary for therapy [81]. The task of a therapist is to encourage the activation of painful memories and to prevent the use of the patient's habitual strategies to avoid or terminate the activation. At the same time, the therapist should support the patient in organizing the declarative memory related to the traumatic event by assisting the narration of the event within the patient's biography. Although the activation of the sensory-perceptual representation leads to a high emotional response, the increasing formation of autobiographical knowledge should lead to an increasing capability by the supervisory structure to inhibit the fear reactions. This effect can be observed as habituation of the emotional response. This principle guides the procedure of NET.

\section{The procedure of narrative exposure therapy}

NET has been developed for application in crisis and postconflict regions. This context poses several challenges for a treatment approach. Because in these countries relatively few professionals are available for a high number of affected people, the treatment must be short and pragmatic. It should be easy to learn and be effective even when provided by trained laypersons or paraprofessionals with no or minimal medical or psychologic backgrounds. It should be applicable across cultures and fit into the social and political background of the setting.

Most researchers who are working with refugees or under field conditions in disaster and war regions are aware that any psychologic intervention needs a firm context. Although PTSD may be the most prevailing mental health disorder in most populations affected by war and disaster, a specific treatment module should be integrated into a sustainable and comprehensive psychosocial or mental health program that includes a variety of interventions and a referral structure for medical conditions. NET can be a key treatment program within such a structure; however, the therapist should be prepared to encounter common conditions, such as ongoing child abuse, substance dependence, grief, and depression. In addition to trauma therapy, therapists should have learned to identify and understand these problems so as to provide assistance or referral to other sources of help.

For the treatment of PTSD, the authors have developed NET, which is based on principles of the theory outlined in the previous sections $[9,26,82]$. For NET, the classical form of trauma exposure therapy was adapted to meet the needs of clinically traumatized survivors of war and torture. The different variants of exposure therapy for PTSD usually target the worst traumatic event, assuming that this approach will lead to the best 
treatment outcome. Most victims of organized violence, war, and torture have experienced several traumatic events, however, and often it is impossible to identify the worst event before treatment. In addition, the fear networks of different traumatic events overlap, and it can be difficult to sort out the origin of different network items. To overcome this challenge, NET uses the chronicity of testimony therapy. Instead of defining a single event as a target for therapy, the patient constructs a narrative of his or her whole life, from birth to the present, while focusing on the detailed report of the traumatic experiences. NET has been adapted for the use with traumatized children and adolescents in a version called "KidNET" [83].

KidNET should not be applied without a formal assessment of the child or adolescent. The typical inclusion criterion for therapy is a diagnosis of PTSD. KidNET has not been studied sufficiently in patients who have current substance addiction and psychosis, so at present these patients should be excluded. KidNET is a short-term treatment approach that usually takes between 4 and 10 sessions of 90 to 120 minutes. Box 1 presents the key

\section{Box 1. Interventions of KidNET in an eight-session format}

\section{Prerequisite}

Structured psychologic assessment including diagnosis of PTSD, comprehensive list of traumatic events, context condition (family background, current threats and violence), and exclusion criteria (substance addiction, psychosis)

First session

Psychoeducation with child and caretaker

Informed consent by caretaker and child

Lifeline

Second session

Revisit lifeline

Start narration at birth

Third through seventh sessions

Revisit lifeline

Reread and correct the narration of the previous session Continue narration with focus on traumatic events

Eighth session

Revisit lifeline and add symbols for hopes in the future Reread whole narration and include hopes for the future Sign and hand over the narration

After treatment

Follow-up examination about 3 months after treatment 
interventions in KidNET as applied in an eight-session treatment. The procedure for KidNET is described in the NET manual [26].

\section{First session: psychoeducation and lifeline}

Any trauma-focused treatment including an exposure element requires high motivation in the child and a good and trustful relationship between the child and the therapist. Consequently, treatment never should be started without detailed psychoeducation of the child. Parental consent should be obtained, if possible, and parents should be included in the psychoeducational part of treatment. The education includes information about trauma symptoms, a simplified model of the memory theory, the implications for treatment, and a detailed explanation of the treatment procedure. The child and the caretaker should be prepared for the activation of memories from the past that might be painful and that they might want to avoid; they should know that the therapist will counteract this tendency. Depending on the child's age, different metaphors can be used to explain the memory theory. (One example is the metaphor of a cabinet that is so full and messy that items fall out of it. Constantly holding the doors and occasionally putting the items back might be one solution, but it would be better to open doors once and to sort the items systematically.) In KidNET, parents or caretakers are present only during the psychoeducational part of treatment. The rest of the treatment is conducted with the child alone (or with an interpreter if necessary), to allow the child to make his or her own report free of the influences of the parents or caretakers.

The next step in treatment is the lifeline procedure. The therapist presents a rope and explains to the child that it symbolizes his or her life, with the beginning of the rope marking the birth. The child is asked to lay the rope out on the floor, leaving a part of the rope unfolded to represent the future. The therapist then presents flowers and stones of different sizes and colors to the child and explains that the flowers represent happy moments in life, and the stones stand for bad experiences (eg, sad, fearful, or painful events). The therapist asks the child to place the flowers and stones along the lifeline to mark significant life events. The therapist assists the child in finding the right chronology of events. Because the aim of the procedure is to obtain a rough overview of the child's life rather than emotional disclosure, the child gives only a very short description - a headlineof each event. After all major events are represented on the lifeline, the child makes a drawing of this lifeline and writes the headline next to each symbol. Older children might prefer to take a photograph of the lifeline instead of drawing. The picture of the lifeline serves as an important tool for knowing where the key moments in the child's life are located. During treatment, more events (more stones and flowers) might be revealed, and the picture can be corrected accordingly. 


\section{The second session}

At the start of each session, the child revisits the picture of the lifeline. Then the child starts to narrate his or her life. The therapist takes care of the chronology of events and starts the procedure with direct questions like "Where were you born?" and "Who are your parents?" The narration should focus both on factual background information and on vividly recorded emotional events. In fortunate cases, the first emotional event can be a positive event that should be explored in much detail, using the same techniques used for traumatic events to teach the child the procedure of chronologic and emotional narration. The therapist writes down the narration as a first-person account in the past tense. The start of a narration of a 12-year-old Sudanese boy could look like this:

I grew up in Yei. We were a large family. My father had four wives by then. And he had 11 children, four girls and seven boys. My father was a rich man. He had goats and cattle. So there was a competition among the children. My father preferred the children of another wife. Me, my brother Nicolas, and my sister Sylvia were among the neglected ones. But I remember well, when we were children, we enjoyed playing in the field while the parents were uprooting cassava.

\section{The following sessions}

At the start of the following sessions the therapist takes some time to explore how the child has been faring between the sessions. This understanding is particularly important, because some children have more intrusive symptoms and nightmares following the initial NET sessions. The therapist explains to the child that this phenomenon is an indication of emotional processing and that the child should not be afraid of this occurrence. After the therapist and the child have indicated the current state of therapy at the lifeline, the therapist asks the child whether he or she wants to modify the lifeline: perhaps the child has remembered additional major events. The therapist then rereads the narration that was written down in the previous session. The therapist instructs the child to be very attentive, to imagine the events again, and to correct the story. Usually, the child adds some information or even new events. The therapist is aware of uncertainties in chronology and resolves these issues with the child. The narration then continues until the current situation.

\section{The last session}

In the last treatment session, the child is asked to lay down his or her lifeline again. This time, the lifeline is extended with flowers to represent the hopes and wishes for the future. The therapist reads the narration to the child one last time and adds the hopes and wishes for the future. At the end, all persons who were involved in the treatment (ie, the child, 
the therapist, and the interpreter) sign the final document. The therapist discusses with the child the further use of the testimony and hands one copy to the child, who is free to do with it whatever he or she wants. The therapist, however, explains the potential dangers involved in politically sensitive cases.

\section{Narrating traumatic events}

The most important and most sensitive element of KidNET is the narration of the traumatic events. Within KidNET, a traumatic event should be narrated only using a very directive approach that requires some skills and a clear understanding of the neurocognitive theory outlined earlier.

Even though most traumatic events should be predictable in the narration, because they are represented as stones on the lifeline, the therapist should be aware of events that turn out to be very dramatic during the narration or events that have been left out on the lifeline. Several indicators show that the child might be approaching a traumatic event in his or her story. The child might show some observable activation of the fear structure (ie, become nervous, tense, and fearful). At the same time, the child might want to avoid the narration by getting impatient and rushing through a period of life or by presenting an urgent need for a break. At this moment the therapist must make a clear decision whether to go explore the event in detail or to postpone the continuation to the next session, because the narration of a traumatic event usually requires about 1 hour and must not be interrupted. As soon as the therapist decides to explore the event, he or she must be very directive in the exploration to keep a clear chronology. Usually, the story is fragmented and dominated by single perceptions of the worst moment of the event. The therapist directs the child to start the event at the beginning, including background information about the temporal and spatial context of the event. The event is then explored in slow motion, in much detail, always keeping the chronology of the event clear. By asking the child direct questions ("What did you see?') and making probing observations ("I can see that you are trembling now. Did you feel like trembling then?'), the therapist makes sure that all aspects of the memory-including "cold" facts as well as "hot" elements from the fear structure-are present at each step of the story. In this way, elements from the fear structure (sensory perceptions, cognitions, emotions, and physiologic responses) are anchored in the framework of a chronologic life event that is based in autobiographic memory. As a consequence, a habituation of emotional responses can be observed during the narration. The child will notice a decrease in fear and perhaps even some feelings of relief. The therapist always should be aware of the increasing and decreasing states of arousal and fear in the child and never should end a session while the child is still highly aroused and occupied by memories of the traumatic event. After the narration of a traumatic event, the child might want to 
discuss some aspects of the meaning of this event. The therapist can use methods from cognitive therapy to modify maladaptive assumptions and conclusions, especially when the child feels high levels of shame and guilt.

\section{The use of illustrative media}

The main tool of narrative exposure is language. Even though autobiographical memory processes, including narrative language, are reasonably well established at the age of 5 years [74], children need more assistance than adults in creating their autobiographic memory, especially for memories that happened before the age of 5 years. Although the child's level of language development at the time of encoding influences the extent to which events can be reported verbally, children at the age of 5 years can provide a brief verbal report of a stressful experience that happened already at the age of 3 years [84]. However, Children, including very young ones, can provide additional information to their verbal report if they are given the opportunity to illustrate their memories [85].

In addition to language development, the child's emotional and cognitive capabilities must be taken into account in trauma treatment [86]. Unlike other treatment approaches for traumatized children, KidNET does not contain a module of training in emotion regulation or affective coping. Nevertheless, the therapist must be aware of the individual child's state of emotional and cognitive development. Most school-aged children can understand at least the basic emotions of fear, anger, sadness, and happiness and can infer the causes of the emotional states [87]. Children's narrations of emotional events usually contain few references to emotions, however [88]. The child-parent communication is the main medium of emotional development. Because child-parent interactions can be severely impaired in traumatized children, many of them present with a marked delay in emotional development.

Because of these developmental factors, the therapist must be aware of the emotional and cognitive capabilities of the individual child. Often the therapist must assist directly in creating an understandable narration. For example, the therapist must be aware of a child's difficulties in classifying and verbalizing emotions and teach the child about basic emotions. Many children have difficulties judging the event that have happened to them, especially in cases of taboo and shameful events such as sexual victimization. A lack of knowledge about children's rights and the context of violence can leave them with a lack of understanding of their own biography. The therapist can educate the child directly about the context of the particular form of violence and can acknowledge the injustice that has happened to him or her.

The use of creative media can compensate for children's difficulties with memory and reporting by providing a chain of retrieval cues, a structure for the child's narrative [89], and assistance in understanding and verbalizing 
emotions [90]. Therapists, however, must be be very cautious not to be suggestive when using illustrative material to avoid the installation of false memories [91].

KidNET involves the use of specific creative media that aid in retrieval of memory and help the narration of a past event. Such tools are the lifeline, drawing, and re-enactment using body positioning or playing out (eg, using little figures or toys) of key moments of the traumatic event. The use of illustrative material always is accompanied by verbal narration. While drawing or playing, the child is encouraged to explain his or her drawing or activity, and the therapist verbalizes along empathically. The dialogue is guided by the rationale of NET treatment: cognitions, emotions, and physiologic and sensory memory within the flow of chronology. For example, the drawing of a scene might be accompanied like this: "I can see a little boy with a red T-shirt by the side of the road here. Is this you? Ah sure, I can recognize your dark hair. Do you remember what you were thinking in that moment as you stood there? How do you feel now you are drawing this?" Body positioning is a different technique that requires the child to assume the position he or she had during the traumatic event. Because the kinesthetic perception is part of the sensory-perceptual representation of the event, this technique might help trigger other hot memories of the event. Because this method can be very powerful in triggering emotions, the therapist should be careful to stay in close contact with the child and to keep assisting in narrating the event.

\section{Basic rules}

The treatment of traumatized children requires several ground rules for the basic behavior and attitude of the therapist. The ground rules (security, control, predictability, physical integrity, acceptance, empathy, congruence, and confidentiality) are explained in more detail in the manual [26]. The main challenge for the therapist is to overcome his or her own avoidance of strong emotions. As a consequence, especially in conflict regions, a processing of the practitioner's traumatic experiences is a key element of NET training.

\section{Outcome of narrative exposure therapy in children}

So far, one case report and two randomized, controlled trials using NET with adults have been published [9,82,92]. In general, these studies with Sudanese refugees in Uganda and with survivors of political violence in Romania showed a clinically significant reduction in PTSD symptoms and greater effectiveness than supportive counseling and psychoeducation only. Treatment effects have been maintained for up to 1 year, and the data show that within the first year after treatment the symptoms continue to decline in comparison with the post-test administered immediately after 
therapy. Three case reports and a small case series on KidNET used to treat war-traumatized children living in a Ugandan refugee camp have appeared in print $[83,93]$. In meantime, controlled studies have been completed. A randomized, controlled trial with traumatized refugee children in Germany showed that children who received KidNET had a large decrease in PTSD in comparison with children on the waiting list. A controlled study with children and adolescents orphaned in the Rwandan genocide demonstrated lasting and rather strong effects. Trials in Sri Lanka proved that KidNET can be disseminated effectively to teachers who then could treat war-traumatized children successfully. Thus there is increasing evidence that KidNET significantly reduces symptoms of PTSD even in severely war-traumatized children. KidNET also seems to have a significant effect on the symptoms of depression and on suicidal ideation. These findings show that KidNET is a feasible, pragmatic, and effective tool for the short-term treatment of child and adolescent victims of war and violence.

\section{References}

[1] Kaldor M. New and old wars: organized violence in a global era. London: Blackwell; 1999.

[2] International Federation of Red Cross and Red Crescent Societies. World disasters report 2004. Geneva: International Federation of Red Cross and Red Crescent Societies.

[3] Catani C, Jacob N, Schauer E, et al. When family violence adds to war and natural disaster: mental health of children living under extreme stress in Sri-Lanka BMC Psychiatry, in press.

[4] Ehntholt KA, Yule W. Practitioner review: assessment and treatment of refugee children and adolescents who have experienced war-related trauma. J Child Psychol Psychiatry 2006;47: 1197-210.

[5] Saigh PA. The development of posttraumatic stress disorder following four different types of traumatization. Behav Res Ther 1991;29:213-6.

[6] Schaal S, Elbert T. Ten years after the genocide: trauma confrontation and posttraumatic stress in Rwandan adolescents. J Trauma Stress 2006;19:95-105.

[7] Elbert T, Huschka B, Schauer E, et al. Trauma-related impairment in children-an epidemiological survey in Sri Lankan provinces affected by two decades of civil war and unrest. Child Abuse Negl, in press.

[8] Neuner F, Schauer E, Catani C, et al. Post tsunami stress: a study of posttraumatic stress disorder in children living in three severely affected regions in Sri Lanka. J Trauma Stress 2006; 19:339-47

[9] Neuner F, Schauer M, Klaschik C, et al. A comparison of narrative exposure therapy, supportive counseling, and psychoeducation for treating posttraumatic stress disorder in an African refugee settlement. J Consult Clin Psychol 2004;72:579-87.

[10] Meiser-Stedman R, Yule W, Smith P, et al. Acute stress disorder and posttraumatic stress disorder in children and adolescents involved in assaults or motor vehicle accidents. Am J Psychiatry 2005;162:1381-3.

[11] Sack WH, Him C, Dickason D. Twelve-year follow-up study of Khmer youths who suffered massive war trauma as children. J Am Acad Child Adolesc Psychiatry 1999;38: $1173-9$.

[12] Bolton P, Bass J, Betancourt T, et al. Interventions for depression symptoms among adolescent survivors of war and displacement in northern Uganda: a randomized controlled trial. JAMA 2007;298:519-27. 
[13] Cohen JA, Deblinger E, Mannarino AP, et al. A multisite, randomized controlled trial for children with sexual abuse-related PTSD symptoms. J Am Acad Child Adolesc Psychiatry 2004;43:393-402.

[14] Stein BD, Jaycox LH, Kataoka SH, et al. A mental health intervention for schoolchildren exposed to violence: a randomized controlled trial. JAMA 2003;290:603-11.

[15] Stallard P. Psychological interventions for post-traumatic reactions in children and young people: a review of randomised controlled trials. Clin Psychol Rev 2006;26: 895-911.

[16] Deblinger E, Steer RA, Lippmann J. Two-year follow-up study of cognitive behavioral therapy for sexually abused children suffering post-traumatic stress symptoms. Child Abuse Negl 1999;23:1371-8.

[17] Smith P, Dyregrov A, Yule W, et al. Children and war: teaching recovery techniques. Bergen, Norway: Children and War Foundation; 2000.

[18] Saltzman WR, Layne CM, Steinberg AM, et al. Developing a culturally and ecologically sound intervention program for youth exposed to war and terrorism. Child Adolesc Psychiatr Clin N Am 2003;12:319-42, x.

[19] Giannopoulou I, Dikaiakou A, Yule W. Cognitive-behavioural group intervention for PTSD symptoms in children following the Athens 1999 earthquake: a pilot study. Clin Child Psychol Psychiatry 2006;11:543-53.

[20] Goenjian AK, Karayan I, Pynoos RS, et al. Outcome of psychotherapy among early adolescents after trauma. Am J Psychiatry 1997;154:536-42.

[21] Chemtob CM, Nakashima JP, Hamada RS. Psychosocial intervention for postdisaster trauma symptoms in elementary school children: a controlled community field study. Arch Pediatr Adolesc Med 2002;156:211-6.

[22] Ehntholt KA, Smith P, Yule W. School-based cognitive-behavioural therapy group intervention for refugee children who have experienced war-related trauma. Clin Child Psychol Psychiatry 2005;10:235-50.

[23] Thabet AA, Vostanis P, Karim K. Group crisis intervention for children during ongoing war conflict. Eur Child Adolesc Psychiatry 2005;14:262-9.

[24] Cienfuegos J, Monelli C. The testimony of political repression as a therapeutic instrument. Am J Orthopsychiatry 1983;53:43-51.

[25] Foa EB, Rothbaum BO. Treating the trauma of rape: cognitive-behavioral therapy for PTSD. Treatment manuals for practitioners. New York; Guilford Press: 1998.

[26] Schauer M, Neuner F, Elbert T. Narrative exposure therapy-a short term intervention for traumatic stress disorders after war, terror or torture. Seattle; Hogrefe: 2005.

[27] Brewin CR. A cognitive neuroscience account of posttraumatic stress disorder and its treatment. Behav Res Ther 2001;39:373-93.

[28] Brewin CR, Holmes EA. Psychological theories of posttraumatic stress disorder. Clin Psychol Rev 2003;23:339-76.

[29] Dalgleish T. Cognitive approaches to posttraumatic stress disorder: the evolution of multirepresentational theorizing. Psychol Bull 2004;130:228-60.

[30] Ehlers A, Clark DM. A cognitive model of posttraumatic stress disorder. Behav Res Ther 2000;38:319-45.

[31] Tulving E. Episodic memory and common sense: how far apart? Philos Trans R Soc Lond B Biol Sci 2001;356:1505-15.

[32] Howe ML, Toth S, Cicchetti D. Memory and developmental psychopathology. In: Cicchetti $\mathrm{D}$, Cohen D, editors. Developmental psychopathology. Developmental neuroscience, vol. 2. 2nd edition. New York: Wiley; 2006. p. 106-25.

[33] Squire LR. Declarative and nondeclarative memory: multiple brain systems supporting learning and memory. In: Schacter DL, Tulving E, editors. Memory systems 1994. Cambridge (MA): MIT Press; 1994. p. 207-28.

[34] Conway MA, Pleydell-Pearce CW. The construction of autobiographical memories in the self-memory system. Psychol Rev 2000;107:261-88. 
[35] Brewin CR, Dalgleish T, Joseph S. A dual representation theory of posttraumatic stress disorder. Psychol Rev 1996;103:670-86.

[36] Metcalve J, Jacobs W. A "hot-system/cool-system" view of memory under stress. PTSD Research Quarterly 1996;7:1-3.

[37] Dudai Y. The neurobiology of consolidations, or, how stable is the engram? Annu Rev Psychol 2004;55:51-86.

[38] Shastri L. Episodic memory and cortico-hippocampal interactions. Trends Cogn Sci 2002;6: $162-8$.

[39] McClelland JL, McNaughton BL, O'Reilly RC. Why there are complementary learning systems in the hippocampus and neocortex: insights from the successes and failures of connectionist models of learning and memory. Psychol Rev 1995;102:419-57.

[40] Conway MA. Sensory-perceptual episodic memory and its context: autobiographical memory. Philos Trans R Soc Lond B Biol Sci 2001;356:1375-84.

[41] Lang P. A bio-informational theory of emotional imagery. Psychophysiology 1979;16: 495-512.

[42] Bremner JD. Neuroimaging studies in post-traumatic stress disorder. Curr Psychiatry Rep 2002;4:254-63.

[43] Kensinger EA. Remembering emotional experiences: the contribution of valence and arousal. Rev Neurosci 2004;15:241-51.

[44] Canli T, Zhao Z, Brewer J, et al. Event-related activation in the human amygdala associates with later memory for individual emotional experience. J Neurosci 2000;20:RC99.

[45] Brown R, Kulik J. Flashbulb memories. Cognition 1977;5:73-99.

[46] Sharot T, Martorella EA, Delgado MR, et al. How personal experience modulates the neural circuitry of memories of September 11. Proc Natl Acad Sci U S A 2007;104:389-94.

[47] LeDoux JE. Emotion: clues from the brain. Annu Rev Psychol 1995;46:209-35.

[48] LeDoux JE. Emotion circuits in the brain. Annu Rev Neurosci 2000;23:155-84.

[49] Miller MM, McEwen BS. Establishing an agenda for translational research on PTSD. Ann N Y Acad Sci 2006;1071:294-312.

[50] Kim JJ, Yoon KS. Stress: metaplastic effects in the hippocampus. Trends Neurosci 1998;21: 505-9.

[51] McEwen BS. Stress and hippocampal plasticity. Annu Rev Neurosci 1999;22:105-22.

[52] Sapolsky RM, Uno H, Rebert CS, et al. Hippocampal damage associated with prolonged glucocorticoid exposure in primates. J Neurosci 1990;10:2897-902.

[53] Pitman RK, Shalev AY, Orr SP. Posttraumatic stress disorder: emotion, conditioning and memory. In: Gazzaniga MS, editor. The new cognitive neurosciences. Cambridge (MA): MIT Press; 2000. p. 72-96.

[54] Duvarci S, Pare D. Glucocorticoids enhance the excitability of principal basolateral amygdala neurons. J Neurosci 2007;27:4482-91.

[55] Debiec J, LeDoux JE. Noradrenergic signaling in the amygdala contributes to the reconsolidation of fear memory: treatment implications for PTSD. Ann N Y Acad Sci 2006;1071: $521-4$.

[56] Vyas A, Mitra R, Shankaranarayana Rao. BS, et al. Chronic stress induces contrasting patterns of dendritic remodeling in hippocampal and amygdaloid neurons. $\mathrm{J}$ Neurosci 2002;22:6810-8.

[57] Williams LM, Phillips ML, Brammer MJ, et al. Arousal dissociates amygdala and hippocampal fear responses: evidence from simultaneous fMRI and skin conductance recording. Neuroimage 2001;14:1070-9.

[58] Foa EB, Kozak MJ. Emotional processing of fear: exposure to corrective information. Psychol Bull 1986;99:20-35.

[59] Brewin CR. Autobiographical memory for trauma: update on four controversies. Memory 2007;15:227-48.

[60] Harvey AG, Bryant RA. A qualitative investigation of the organization of traumatic memories. Br J Clin Psychol 1999;38:401-5. 
[61] Jones C, Harvey AG, Brewin CR. The organisation and content of trauma memories in survivors of road traffic accidents. Behav Res Ther 2007;45:151-62.

[62] Shin LM, Orr SP, Carson MA, et al. Regional cerebral blood flow in the amygdala and medial prefrontal cortex during traumatic imagery in male and female Vietnam veterans with PTSD. Arch Gen Psychiatry 2004;61:168-76.

[63] Shin LM, Wright CI, Cannistraro PA, et al. A functional magnetic resonance imaging study of amygdala and medial prefrontal cortex responses to overtly presented fearful faces in posttraumatic stress disorder. Arch Gen Psychiatry 2005;62:273-81.

[64] Milad MR, Quirk GJ. Neurons in medial prefrontal cortex signal memory for fear extinction. Nature 2002;420:70-4.

[65] Quirk GJ, Gehlert DR. Inhibition of the amygdala: key to pathological states? Ann N Y Acad Sci 2003;985:263-72.

[66] Quirk GJ, Likhtik E, Pelletier JG, et al. Stimulation of medial prefrontal cortex decreases the responsiveness of central amygdala output neurons. J Neurosci 2003;23:8800-7.

[67] Santini E, Ge H, Ren K, et al. Consolidation of fear extinction requires protein synthesis in the medial prefrontal cortex. J Neurosci 2004;24:5704-10.

[68] Hariri AR, Mattay VS, Tessitore A, et al. Neocortical modulation of the amygdala response to fearful stimuli. Biol Psychiatry 2003;53:494-501.

[69] Ochsner KN, Bunge SA, Gross JJ, et al. Rethinking feelings: an FMRI study of the cognitive regulation of emotion. J Cogn Neurosci 2002;14:1215-29.

[70] Phan KL, Fitzgerald DA, Nathan PJ, et al. Neural substrates for voluntary suppression of negative affect: a functional magnetic resonance imaging study. Biol Psychiatry 2005;57:210-9.

[71] Phelps EA. Emotion and cognition: insights from studies of the human amygdala. Annu Rev Psychol 2006;57:27-53.

[72] Howe ML. The fate of early memories: developmental science and the retention of childhood experiences. Washington; American Psychological Association.

[73] Brainerd CJ, Reyna VF. Fuzzy-trace theory: dual processes in memory, reasoning, and cognitive neuroscience. Adv Child Dev Behav 2001;28:41-100.

[74] Nelson K, Fivush R. The emergence of autobiographical memory: a social cultural developmental theory. Psychol Rev 2004;111:486-511.

[75] Teicher MH, Andersen SL, Polcari A, et al. Developmental neurobiology of childhood stress and trauma. Psychiatr Clin North Am 2002;25:397-426, vii-viii.

[76] Scheeringa MS, Zeanah CH, Myers L, et al. New findings on alternative criteria for PTSD in preschool children. J Am Acad Child Adolesc Psychiatry 2003;42:561-70.

[77] Scheeringa MS, Salloum A, Arnberger RA, et al. Feasibility and effectiveness of cognitivebehavioral therapy for posttraumatic stress disorder in preschool children: two case reports. J Trauma Stress 2007;20:631-6.

[78] Kenardy J, Smith A, Spence SH, et al. Dissociation in children's trauma narratives: an exploratory investigation. J Anxiety Disord 2007;21:456-66.

[79] Carrion VG, Weems CF, Reiss AL. Stress predicts brain changes in children: a pilot longitudinal study on youth stress, posttraumatic stress disorder, and the hippocampus. Pediatrics 2007;119:509-16.

[80] Foa EB, Molnar C, Cashman L. Change in rape narratives during exposure therapy for posttraumatic stress disorder. J Trauma Stress 1995;4:675-90.

[81] Jaycox LH, Foa EB, Morral AR. Influence of emotional engagement and habituation on exposure therapy for PTSD. J Consult Clin Psychol 1998;66:185-92.

[82] Neuner F, Schauer M, Elbert $T$, et al. A narrative exposure treatment as intervention in a Macedonia's refugee camp: a case report. J Behav Cogn Psychother 2002;30:205-9.

[83] Onyut LP, Neuner F, Schauer E, et al. Narrative exposure therapy as a treatment for child war survivors with posttraumatic stress disorder: two case reports and a pilot study in an African refugee settlement. BMC Psychiatry 2005;5.

[84] Peterson C, Rideout R. Memory for medical emergencies experienced by 1- and 2-year-olds. Dev Psychol 1998;34:1059-72. 
[85] Driessnack M. Children's drawings as facilitators of communication: a meta-analysis. J Pediatr Nurs 2005;20:415-23.

[86] Salmon K, Bryant B. Posttraumatic stress disorder in children-the influence of developmental factors. Clin Psychol Rev 2002;22:163-88.

[87] Ashiabi GS. Promoting the emotional development of preschoolers. Early Child Educ J 2000;28:79-84.

[88] Fivush R. Children's memories of emotional events. In: Reisberg D, Hertel P, editors. Memory and emotion. Oxford (UK): Oxford University Press; 2003. p. 242-71.

[89] Gross J, Hayne $\mathrm{H}$. Drawing facilitates children's verbal reports of emotionally laden events. J Exp Psychol Appl 1998;4:163-79.

[90] Wesson M, Salmon K. Drawin and showing: helping children to report emotionally laden events. Appl Cogn Psychol 2001;15:301-20.

[91] Brown DA, Pipe ME, Lewis C, et al. Supportive or suggestive: do human figure drawings help 5- to 7-year-old children to report touch? J Consult Clin Psychol 2007;75:33-42.

[92] Bichescu D, Neuner F, Schauer M, et al. Narrative exposure therapy for political imprisonment-related chronic posttraumatic stress disorder and depression. Behav Res Ther 2007;45: $2212-20$.

[93] Schauer E, Neuner F, Elbert T, et al. Narrative exposure therapy in children: a case study. Intervention 2004;2:18-38. 